\title{
POR UN ADULTO SANO POLIMORFO
}

\author{
Carlos Giménez Lorente ${ }^{1}$ \\ Institut Català D. Winnicott, IPR, FEAP
}

Durante siglos, la sexualidad del ser humano en Occidente ha sido instrumentalizada para la normalización de ciudadanos. Se han institucionalizado unas prácticas sexuales garantes de los intereses políticos, religiosos y económicos de las élites. Ante estos dispositivos reguladores que naturalizan un patrón heterosexual-genital-monógamo-identitario de la sexualidad humana, conviene plantearse si la Clínica participa como un eslabón más de la cadena productiva de sujetos o, por el contrario, puede subvertir el dispositivo normalizador con enfoques más abiertos y plurales. Perfilar una realidad sexual más amplia, es posible. En mi propuesta, defiendo una corporeidad extensamente erógena, un posicionamiento identitario más integrador de sexo y género, y una relacionalidad amplia en cuanto a objetos-sujetos de deseo. En definitiva, anticipo un nuevo adulto polimorfo sano, no necesariamente inmaduro, como resultado de una política más extensiva e inclusiva del deseo.

Palabras clave: sexualidad, perversión, normalización, monogamia, intersubjetividad, animalidad

For centuries human sexuality in the West has been instrumentalised towards normalisation. Sexual practices have been institutionalised to guarantee the elite's political, religious and economic interests. When considering these regulatory mechanisms, which naturalise a heterosexual-genitalmonogamous version of human sexuality, an important question should be raised: is clinical work one more link in the chain of producing subjects, or can it subvert normalising mechanisms by offering perspectives that are more open and diverse. Defining a more encompassing notion of sexual reality is possible. I defend an extensively erogenous corporeality, a more integrative position towards sexual and gender identity, and a broader way of relating as far as objects-subjects of desire are concerned. In sum, I foresee a new healthy polymorphous adult - not one who is necessarily immature -as a result of a politics that is both more wide-ranging and inclusive in terms of desire.

Key Words: sexuality, perversion, normalisation, monogamy, intersubjectivity, animality.

English Title: FOR A HEALTHY, POLYMORPHOUS ADULT

\section{Cita bibliográfica / Reference citation:}

Giménez Lorente, C. (2018). Por un adulto sano polimorfo. Clínica e Investigación Relacional, 12 (3): 556-567. [ISSN 1988-2939] [Recuperado de www.ceir.info ] DOI: 10.21110/19882939.2018.120309

\footnotetext{
${ }^{1}$ Psicólogo y Psicoterapeuta acreditado FEAP, Miembro del Institut Català D. W. Winnicott (Barcelona), del Instituto de Psicoterapia Relacional (Madrid). Dirección de contacto: cgimenezlo@gmail.com
} 
Como terapeuta siempre me ha inquietado si el ejercicio de esta profesión actúa como un dispositivo normalizador de ciudadanos. Y la sexualidad, en su abordaje en sesión, puede ser un campo especialmente abonado para esta normalización, porque como reducto de lo supuestamente "natural", legitima todavía más el dispositivo.

Las prácticas psicológicas, como cualquier práctica social, no dejan de ser una tecnología de producción de verdad.

Con este artículo, no pretendo desvelar verdades ocultas, sino contribuir a perfilar algunas nuevas, a construirlas en el ámbito específico de la sexualidad. Habituadas a apropiarse de las prácticas de subjetividad, las élites de la política, la religión, la clínica o el mercado, se han ocupado sobradamente de esta labor.

Obviamente, cualquier verdad ad hoc tampoco es posible, ya que la hermenéutica tiene sus límites. La cuestión que nos ocupa es hasta dónde podemos extender los límites de la interpretación-construcción de la sexualidad.

Teniendo en cuenta que estamos constituidos biográficamente por esas prácticas normalizadoras, también asumo la conveniencia de respetar "de alguna manera" esas verdades inoculadas sin dejar de desafiarlas.

Desde el paradigma del Psicoanálisis Relacional, damos por descartada la neutralidad del terapeuta. Como apunta Francesc Sáinz, no hay nada menos neutral que un terapeuta que pretende ser neutral. En opinión de Joan Coderch (2001), el terapeuta no puede evitar imponer su método de trabajo, su práctica terapéutica, incluso debemos aceptar la carga de sugestión que llevan implícitas sus intervenciones.

Así pues, si no es posible ser neutral al tratar la sexualidad del paciente, la concepción que tengamos de un sujeto sexualmente sano y la voluntad de ayudarle en este campo serán transmitidas al paciente, explícita o implícitamente, con nuestras intervenciones verbales, con nuestra gestualidad y siempre con la colaboración de las neuronas espejo, tan sensibles a la intencionalidad del otro, en este caso, del terapeuta.

Admitiendo esta ausencia de neutralidad, urge comprender la dimensión histórica de la sexualidad previa al encuentro con el paciente. De los relatos que se desprenden de nuestro pasado, se edifican nuestras creencias del presente.

Veamos, someramente, qué se ha entendido por sexualidad en la historia de Occidente y quién es ese sujeto sexualmente sano, o no, que nos proponen algunos autores desde la filosofía (Comte-Sponville, 2012) o el psicoanálisis. 
Platón propone en El Banquete no fiarse del deseo porque quien se le entrega, "solo quiere llenarse de él, sin dar ningún valor al carácter del alma del amado".

San Agustín, en sus Confesiones no ve más que inmundicia, podredumbre y soberbia abyección en la sexualidad.

Spinoza en el Tratado de la reforma del entendimiento se refería al arrebato del espíritu por el placer sexual, el cual "le impide del todo pensar en otro bien", y cómo la "tristeza extrema" sigue al "placer de la voluptuosidad".

Montaigne, más abierto al goce, no puede aceptar una sexualidad sin amor: "Me horroriza imaginar que es mío un cuerpo privado de afecto (...) se ama un cuerpo sin alma cuando se ama un cuerpo sin su consentimiento y sin su deseo".

Schopenhaver pone la sexualidad al servicio de la especie y apunta que la naturaleza "aguijonea con todas sus fuerzas al hombre, al igual que al resto de los animales, para que se reproduzca".

Kant se ve obligado a aceptar la naturaleza "bestial" de la sexualidad al servicio de la supervivencia, pero pretende hacerla moralmente aceptable, humanizarla.

Nietzsche, muy crítico con el cristianismo y el judaísmo, reivindica el valor del cuerpo, en tanto que cuerpo sexuado. Para él, el cuerpo es lo primero, pues no existe nada más. Por eso la sexualidad precede a la afectividad, a la que hace posible y a la que engendra. $Y$ sobre la voluptuosidad y para contrarrestar el complot histórico contra el cuerpo, añade en Así habló Zaratustra: "algo inocente y libre para corazones libres, la felicidad del jardín terrenal, el desbordante agradecimiento de todo futuro al ahora".

¿Qué significados recogemos de todas estas aportaciones? La sexualidad como fuerza imparable, como amenaza para la sociedad, como instinto indisociable de la naturaleza, como comportamiento animal destinado a la reproducción y como conducta vergonzosa que solo puede amansar el amor. Siempre hay excepciones, obviamente, como Nietzsche, que apunta en otra dirección con una crítica feroz de la moral cristina que le lleva a reivindicar la voluptuosidad.

Freud produce una ruptura epistemológica de gran alcance al desligar la construcción psíquica de la sexualidad, de la diferencia anatómica. El impulso irrefrenable de la excitación delega en lo psíquico la elaboración de un mapa de fantasmas con los que el sujeto representa la realización del deseo. Ese mapa configura un cuerpo erógeno que no coincide con el anatómico. En sus propias palabras: "en realidad el cuerpo entero es una zona erógena" (Tubert, 2000, pág. 99). 
No por ello, Freud deja de naturalizar la sexualidad. Por un lado, cree que se origina en las funciones vitales, aunque se desprenda de ellas tempranamente $y$, por otro, con la llegada de la pubertad, parece que lo genital retoma la diferencia anatómica esencial entre hombres y mujeres para constituir el niño "sano", antes "perverso polimorfo".

En sus estudios sobre la sexualidad, la pulsión sexual, con todo su empuje, su variedad de fuentes y objetos, acaba tras la pubertad al servicio de la genitalidad y la procreación. Cualquier desvío de este fin se considerará perversión.

El concepto de perversión se asoció desde la psiquiatría clásica al concepto de anormalidad en el comportamiento sexual. $Y$ no solo anormalidad bajo el criterio estadístico, o sea, un comportamiento inusual, poco frecuente, sino anormalidad, como conducta perturbada, desadaptada, patológica y/o insana.

Uno se pregunta, ¿por qué se vinculó con tanta fuerza la perversión con las prácticas sexuales, en lugar de, por ejemplo, con tantas prácticas políticas orientadas a dañar a diferentes colectivos humanos? En mi opinión, la sexualidad era y es un candidato ideal para la normalización en el sentido de que se puede naturalizar con facilidad, es común a todos los seres humanos y posee una carga emocional fuera de dudas. Así pues, todas aquellas conductas sexuales "perversas" que no contribuían a sostener la pareja heterosexual procreadora, quedaron fuera de la normalidad.

Todas las culturas tienen su propia concepción de la normalidad/anormalidad, bajo criterios biológicos, socioculturales, estadísticos o subjetivos. Difícilmente podemos renunciar a este binario, pero sí podemos redefinir y combinar sus criterios ante una realidad compleja que nos interpela. Estudiar esta realidad no debería dirigirse a normalizar un estilo de vida y excluir el resto.

No es conveniente utilizar los mismos criterios de normalidad para todo el abanico de conductas del ser humano. Se puede valorar la anormalidad patológica del autista bajo el criterio estadístico, por poco frecuente, bajo el biológico, en cuanto a la susceptibilidad genética en su etiología, y bajo el legal por su grado de discapacidad.

Sin embargo, una persona con preferencias por el sexo anal o las prácticas sadomasoquistas consentidas, no será adecuado valorar su anormalidad bajo los mismos criterios. Se deberán priorizar otros, como el criterio subjetivo, que atiende a la percepción de bienestar o malestar que experimenta el propio sujeto con su conducta.

Tampoco debemos ser tan ingenuos como para pensar que la percepción del sujeto de su conducta sexual no está condicionada por el criterio sociocultural, o sea, todo aquello regulado por los códigos culturales de su habitat. Es aquí, en los códigos culturales, 
donde debemos incidir más para generar nuevos consensos $y$, con ello, nuevas subjetividades que puedan transitar desde la anormalidad patológica a la normalidad saludable.

Veamos algunas aportaciones históricas sobre la perversión sexual.

Muchos psicoanalistas descendientes de Freud, de corte kleiniano o poskleiniano, como Donald Meltzer, Hanna Segal o la propia Melanie Klein, secundan y perpetúan el dispositivo normalizador y patologizador de la sexualidad sana y perversa, respectivamente. Estos autores creen descubrir en qué consiste la 'buena sexualidad', cuando a mi entender, solo sustentan la moral restrictiva de su época.

Meltzer afirma que las relaciones sexuales de los homosexuales permanecen estancadas al nivel de los juegos previos y al nivel infantil y/o perverso (O'Connor y Ryan, 2003). Para él, un ejemplo típico de la confusión zonal se haya entre la lengua y el pene para el varón y la boca y la vagina para la mujer. Cuando se unen las confusiones entre lo bueno y lo malo y entre lo femenino y lo masculino, el adolescente no sabe distinguir entre la sexualidad buena y la perversa. Está confundido entre la parte contraria a su sexo y la homosexualidad perversa (Meltzer y Harris, 2011).

Hanna Segal sostiene que las lesbianas están atrapadas en una fantasía de ataque envidioso a la pareja heterosexual y que el fracaso para lograr la heterosexualidad es, como tal, la incapacidad de comprender la realidad (O'Connor y Ryan, 2003).

Y para Melanie Klein la homosexualidad también es un fracaso del desarrollo, la designa como un conflicto edípico negativo no resuelto (O'Connor y Ryan, 2003).

Las contribuciones de otros psicoanalistas del Grupo Independiente tampoco difieren mucho. Para Kohut y Winnicott, las perversiones siguen siendo un fracaso del desarrollo. El primero relaciona el placer perverso con la carencia estructural y con la fallida integración del self. El segundo atribuye este fracaso del desarrollo y de la integración del self a una hipoestimulación del niño abandonado o a una hiperestimulación del niño idolatrado (Castaño, 2011). Lo que no quiere decir necesariamente que estos dos autores atribuyan, por ejemplo, a la homosexualidad, la condición inherente de perversidad.

Erich Fromm en El arte de amar nos advierte del peligro de entregarse al sexo genital sin amor:

"La búsqueda del orgasmo sexual asume un carácter que lo asemeja bastante al alcoholismo o la afición a las drogas. Se convierte en un desesperado intento de escapar a la angustia que engendra la separatidad y provoca una sensación cada vez mayor de 
separación, puesto que el acto sexual sin amor nunca elimina el abismo que existe entre dos seres humanos, excepto de forma momentánea" (Fromm, 2003, p.27).

Psicoanalistas relacionales más contemporáneos, como Stephen Mitchell, enfatizan el valor del goce sexual: "el disfrute de la sexualidad como algo animal, ser animales juntos puede implicar el uso mutuo de cada uno, logrando con ello una claridad e inmediatez que acaso no se encuentre en la sutil coreografía de otras dimensiones de la intimidad" (Mitchell, 1993, p.85)

Para finalizar este breve e incompleto repaso histórico, incluyo la definición del concepto de perversión del Diccionario de Psicoanálisis de Laplanche y Pontalis (1993, p.272): "desviación con respecto al acto sexual 'normal', definido como coito dirigido a obtener el orgasmo por penetración genital, con una persona del sexo opuesto". Claro y conciso.

A mi entender, estas concepciones de la sexualidad sana y perversa todavía siguen muy vigentes, quizá no tanto como genitalidad exclusivamente al servicio de la reproducción, pero sí, genitalidad como zona erógena preminente y dirigida al encuentro heterosexual. Aun así, no podemos negar la existencia de otros discursos contemporáneos, todavía subalternos, que han cuestionado el dispositivo normalizador y han propuesto una nueva sexualidad más abierta y gozosa.

Foucault, utilizando el método genealógico de Nietzsche, rastrea la historia de la sexualidad concebida como producto de las fuerzas sociales de cada tiempo (Foucault, 2009).

Según Foucault, hubo un primer régimen de lo lícito-ilícito bajo el poder soberano, fundamentado en la ley y el matrimonio, como dispositivos diseñados para garantizar la economía, que no el amor, y los derechos de herencia. El sodomita era un criminal que intentaba esquivar la ley.

Posteriormente, se instaura el régimen de la sexualidad bajo el poder biopolítico, basado en el binomio normal-anormal. El sujeto interioriza estas ideas, que regularán la conducta con más eficacia que la ley, porque fluyen de la disposición interna de uno, de la identidad. A mediados del siglo XIX nace el homosexual como especie anormal, que más que esquivar la ley, se avergüenza de su naturaleza perversa.

El despliegue de la sexualidad se da con los diferentes dispositivos de confesión, hacer hablar a los deseos facilita su control. Primero, la confesión para exorcizar los "deseos impuros" de los propios monjes y más tarde la confesión de feligreses, la confesión al 
psiquiatra, al psicólogo, al maestro, al padre o al juez. Y no solo se exteriorizan los deseos íntimos, sino que se contabilizan y se miden bajo el paraguas de la ciencia.

Paul B. Preciado en su Testo Yonqui (2008) recoge y desarrolla el legado de Foucault. Visibiliza un nuevo régimen sexual que se solapa con los anteriores, constituyendo un crisol de diferentes niveles de control social que operan en diferentes frentes.

Es el régimen farmacopornográfico. Nuevos dispositivos normalizadores emergen desde la Clínica a mediados del siglo XX con la invención de la píldora anticonceptiva, seguida de la cirugía de reasignación de género, el Viagra y la cirugía estética de los genitales. Todos ellos, dispositivos cuyas prácticas se ejercen, una vez más, en torno a los genitales, órgano hegemónico de regulación social.

Por su parte, la omnipresente industria pornográfica se ha impuesto en lo cotidiano, economizando unas prácticas sexuales muy falocéntricas, y a menudo, imposibles de ejecutar.

¿Necesitamos más evidencias del apropiamiento de nuestro sexo/género, incluso de nuestra biología, por parte de la Clínica, los medios de comunicación o las recientes políticas identitarias (gay, hetero, trans, bisexual, intersexual o lesbiana)?

Yo me sumo a reformular y desnaturalizar estas concepciones, a esbozar una nueva realidad sexual menos reduccionista en cuanto a las fuentes erógenas, a la elección de objetos-sujetos de placer y a la identidad sexual. En definitiva, anticipo un nuevo adulto polimorfo sano, no necesariamente inmaduro, como resultado de una política más extensiva e inclusiva del deseo.

En cuanto a las fuentes erógenas, apuesto por recuperar y mantener aquel goce temprano de nuestros cuerpos sexuados, junto a las posibilidades de una genitalidad que no tiene por qué ostentar todo el poder del erotismo. Propongo una reerotización del ano, los pezones, las axilas, los pies, la boca y/o la piel en su totalidad, incluyendo objetos externos como los Ilamados juguetes sexuales, que agregan nuevas potencialidades al sujeto-cyborg.

Aun admitiendo un desarrollo sexual donde la genitalidad hace su entrada al final de un proceso, todos los estadios anteriores con sus zonas erógenas correspondientes no tienen necesariamente que quedar ni inhibidos en ese momento del desarrollo ni patologizados por regresión-fijación de la libido. Es posible una sexualidad acumulativa, donde los pesos de cada fuente orgánica (oral, anal...), aunque vayan variando en el desarrollo sexual, puedan potenciarse en la vida adulta sin excluirse entre ellos. 
La inclusión de la tecnología en las prácticas sexuales (dildos, aplicaciones online de contactos o farmacología) nos plantea un sujeto híbrido que se constituye y se potencia con estos dispositivos. La tecnología no sustituye ni atrofia necesariamente las capacidades humanas, versión pesimista de la tecnología, sino que puede desplegar capacidades de goce y comunicación.

Quizá, lo humano ronda entre lo tecnológico y lo animal. La sexualidad, en no pocas ocasiones, nos recuerda ese animal interior que tiende a relaciones cosificadas cuando está follando, permítanme la grosería. Ese animal también nos constituye como humanos. La compenetración animal-humano genera el erotismo que no solo trasciende la sexualidad mecánica, sino que antepone la ley del deseo a la ley del placer.

La reerotización de este cuerpo humano-animal-tecnológico entronca con un saber corporal que ya nos proponía Schopenhaver. A su tesis principal (Bengoechea, 2017), "el mundo es mi representación", en la que afirmaba que el ser humano es un ser cognoscente que no puede salir de sí mismo para acceder a la realidad, añade una excepción muy relevante para el tema que nos ocupa.

Propone que hay todo un mundo no representativo de apetitos, de descargas emocionales como el placer, que proporcionan un saber del cuerpo mucho más inmediato e íntimo que la percepción sensorial. Como si hubiera dos cuerpos, el percibido y el sentido. En relación a estas aportaciones, considero porque que el goce sexual vivifica el cuerpo de una forma inmediata y lo acompaña de una carga de existencia que no necesita de mucha elaboración mental.

Esta reivindicación del cuerpo sintiente, distanciado de las representaciones mentales, sintoniza con la visión de Winnicott en su trabajo "La mente y su relación con el psiquesoma" (1949), donde el autor nos previene de un funcionamiento hipertrófico de la mente del niño si no se integra con las emociones y las sensaciones corporales. Entregarse en confianza al otro, supone, dejar "en reposo" la mente (Sáinz, 2017).

Respecto a la reformulación del objeto-sujeto de placer, allí donde se dirige nuestro deseo, Freud ya contribuyó a delinear un mapa extenso, aunque limitado esencialmente a la infancia. Definió el objeto de la pulsión sexual como lo que se incorpora imaginariamente (Tubern, 2000), y la imaginación es muy prolífica. De hecho, tuvo el atrevimiento, para su época, de afirmar que los seres humanos somos potencialmente bisexuales.

Recientes investigaciones confirman esta tesis de Freud (Rieger, G., SavinWilliams, RC., Chivers ML., y Bailey, J.M., 2016), en cuanto que existe un continuo en la 
orientación sexual donde los sujetos no se ubican exclusivamente en los extremos (atracción heterosexual-homosexual) sino en algún punto intermedio.

Los psicólogos tendemos a disponer la sexualidad del paciente al servicio de sus relaciones íntimas, amorosas, significativas e intersubjetivas. No voy a negar que yo también soy partidario de este modo de relación, pero me resisto a reducirla a esta única manera de ejercerla saludablemente.

Es evidente que, a lo largo de la historia, se ha sobredimensionado la importancia de la sexualidad en la vida de los seres humanos, sea porque garantizaba la continuidad de la especie o porque aseguraba la continuidad del dispositivo familia para el orden social. La monogamia, actualmente, monogamia seriada (diferentes parejas, pero no simultáneas), se naturalizó limitando las múltiples expresiones del deseo.

En nuestro quehacer diario, nos involucramos en multitud de relaciones laxas, "interobjetivas", en cuyas interacciones nos cosificamos los unos a los otros. $Y$ precisamente nos cosificamos, porque nos reconocemos como sujetos, aunque no nos ofrezcamos como tales en ese momento concreto. No se puede cosificar un objeto, se cosifican los sujetos.

Las relaciones sexuales pueden darse gozosamente sin necesidad de tanta subjetividad ni intimidad. Obviamente, las sensaciones corporales pueden elicitar afectos, pero no es imprescindible el amor como 'director de orquesta'. Incluso, una interacción sexual cosificada puede ser el preludio, o no, de una relación intersubjetiva mucho más íntima y amorosa.

Por otra parte, creo que es una ilusión pretender relacionarse con el "otro" como un verdadero "otro-sujeto", sobre todo en las primeras interacciones sexuales. Como apunta Mitchell, es muy probable que el "otro" solo represente otra forma del propio self, a menudo, el opuesto:

"Y como el self se define tan centralmente en términos de no-self, la alteridad, el tipo de alteridad que dispara la pasión erótica, podría considerarse una forma de sí mismo, una imagen especular" (Mitchell, 2002, pág.82).

Respecto a la identidad sexual o de género, seguimos atrapados en los binarios, hombremujer (categorías sexuales), femenino-masculino (categorías de género). Sabemos que formamos parte de una trama cultural con prácticas rituales que nos constituyen; por lo tanto, ¿no serán ficciones? Las identidades en general, y la sexual en particular, nos violentan de alguna manera, nos exigen un papel al que a menudo cuesta acomodarse. Podemos deconstruirlas, podemos desdibujar los géneros impuestos, pero también 
podemos reasignar e integrar aquello que se ha atribuido por separado a cada género, a un mismo sujeto, un nuevo sujeto.

Winnicott estudia qué hay de masculino, entendido como el hacer, y de femenino, entendido como el ser, en los hombres y las mujeres. Admite la predisposición a la bisexualidad (Sáinz, 2017) y la conveniencia de que puedan complementarse estos atributos en un mismo sujeto, sean del género que sean.

Es de agradecer, a los activistas LGTBI, al transfeminismo y a la teoría Queer, las nuevas aportaciones que deconstruyen los esencialismos respecto a la identidad sexual y afirman la autodesignación de la identidad, más allá de las categorías sexuales o de género establecidas.

No me gustaría que se confundiera esta apertura de horizonte político de transformación con una defensa de la sociedad pornográfica o de la sociedad de consumo sexual. No se trata de promover una excitación permanente o un estado deseante que nunca se aplaca. De hecho, la pornografía clásica, con su exceso de exhibición, destruye la sexualidad y el erotismo con mayor eficacia que la represión. Capítulo aparte merecen las nuevas propuestas de la pornografía feminista-ética que abogan por una sexualidad igualitaria, erótica y mucho más realista.

Mi propuesta es una sexualidad desacralizada, gozosa y sostenida tanto por una corporeidad extensamente erógena, como por una relacionalidad amplia, no supeditada necesariamente ni al amor ni a la monogamia, pero sí al respeto y consentimiento mutuo.

Quizá conviene señalar, aunque solo sea a modo de presentimiento, la relación entre Eros, amor y sexualidad. Byung-Chul Han sostiene que si el amor deviene solo sexualidad, el Eros se aleja (2017).

Yo considero, en contra de esta tesis, que la sexualidad sin amor puede ser muy erótica si no se imponen interacciones precipitadas que no se prestan a un cierto juego enigmático. Si bien, por otro lado, la sexualidad en sí misma, puede ser suficiente como experiencia de goce sin la visita de Eros.

En cuanto al amor, agotado el estado de enamoramiento, éste se va despidiendo inevitablemente de la seducción de Eros porque el misterio y la transgresión no conjugan bien con el amor duradero. También en este caso, la experiencia amorosa, incluso sin apenas sexualidad, puede ser suficientemente satisfactoria sin la concurrencia de Eros.

A lo largo de este artículo he intentado cuestionar los binarios que tan a menudo constriñen la sexualidad: hombre-mujer, masculino-femenino, homosexual- 
heterosexual, normalidad-anormalidad o humano-animal. Categorías que delimitan unas fronteras que claman por ser transitadas por caminos compartidos de ida y vuelta, caminos que en lugar de comprometer o enfrentar la multiplicidad de identidades que nos habitan, las enriquecen.

Quiero puntualizar también que estas ideas no son solo resultado de la palabra escrita (libros, artículos...) o de la palabra hablada (amigos, profesores, pacientes, familia...), sino también de mi propia experiencia sexual. Porque, ¿acaso no sería banalizar este trabajo si no lo hubiera encarnado en mi cotidiano?

\section{REFERENCIAS}

Bailey, J.M., Rieger, G., Savin-Williams, RC. y Chivers ML., (2016). Sexual Arousal and Masculinity-Feminity of Women. Journal of Personality and Social Psychology, No.2: 265-283

Bengoechea, F. (2017, Octubre-Diciembre). Signos de vida. Nueva visita a la filosofía de Nietzsche. Seminario llevado a cabo por el Institut d'Humanitats en el Centre de Cultura Contemporànea de Barcelona.

Byung-Chul, H. (2017). La agonía del Eros. Barcelona: Herder.

Castaño, R. (2011). La terapia sexual. Una mirada relacional. Madrid: Col. Pensamiento relacional $\mathrm{n}{ }^{\circ} 4$. Ágora Relacional.

Coderch, J. (2001). La relación paciente-terapeuta. El campo del psicoanálisis y la psicoterapia psicoanalítica. Barcelona: Fundació Vidal i Barraquer - Paidós.

Comte-Sponville, A. (2012). Ni el sexo ni la muerte. Tres ensayos sobre el amor y la sexualidad. Barcelona: Paidós.

Foucault, M. (2009). La historia de la sexualidad. vol. 1. La voluntad del saber. Madrid: Siglo XXI.

Fromm, E. (2003). El arte de amar. Barcelona: Col. Contextos. Paidós.

Laplanche, J. y Pontalis, J.B. (1993). Diccionario de psicoanálisis. Barcelona: Paidós.

Meltzer, D y Harris, M. (2011). Adolescence. Talks and papers by Donald Meltzer and Martha Harris. Londres: Karnac.

Mitchell, S.A. (2002). Can love last? The fate of romance over time. New York. W.W. Norton \& Company.

O'Connor, N. y Ryan, J. (2003). Wild desires and mistaken identities. Lesbianism and psychoanalysis. Londres: Karnac.

Preciado, B. (2008). Testo Yonqui. Madrid: Espasa.

Sáinz, F. (2017). Winnicott y la perspectiva relacional en el psicoanálisis. Barcelona: Col. Salud Mental. Fundació Vidal i Barraquer - Paidós.

Tubert, S. (2000). Sigmund Freud. Fundamentos del psicoanálisis. Madrid: EDAF. 
Winnicott, D.W. (1949). Mind and its relation to be Psyche-Soma. en Collected Papers. Through Paediatrics to Psycho-Analysis. Londres: Tavistock Publications; Nueva York, Basic Books, 1958 [trad. cast.: "La mente y su relación con el psique-soma", en Escritos de pediatría y psicoanálisis. Barcelona: Paidós, 1998, pp. 325-340].

Original recibido con fecha: $\quad 3 / 6 / 2018 \quad$ Revisado: 30/9/2018 Aceptado: 30/10/2018 